\title{
The Debt Choices of the Firms in Developed Countries: Evidence from G-7
}

\author{
Pasquale De Luca ${ }^{1}$ \\ ${ }^{1}$ Faculty of Economics, "Sapienza" University, Rome, Italy \\ Correspondence: Pasquale De Luca, Faculty of Economics, "Sapienza" University, Rome, Italy. Tel: \\ 39-06-4976-6454. E-mail: pasquale.deluca@uniroma1.it \\ Received: January 19, 2015 \\ Accepted: February 16, 2015 \\ Online Published: March 25, 2015 \\ doi:10.5539/ijef.v7n4p122 \\ URL: http://dx.doi.org/10.5539/ijef.v7n4p122
}

\begin{abstract}
This study examines the main determinants that affect the firm's debt choices. Based on a panel data of non-financial listed firms in G-7 countries in the period 1994-2013, the study shows that the firm's choices about debt level are functions of several previsions over time about, on the one hand, to the firm's characteristics and its expected performances and, on the other hand, to the economic, financial and institutional system of country's reference.
\end{abstract}

Keywords: capital structure, debt level, leverage, debt capacity

\section{Introduction}

The firm's debt level choice is one of the most relevant problems for managers and financial economists. It is still open despite relevant theoretical literature and decades of empirical tests.

Modigliani-Miller's theory $(1958,1963)$ is considered the starting point of the modern theory of the capital structure. They argue that capital structure choices are irrelevant both on the value of the firm than on its cost of capital (Proposition I and II). Over the years, removing some restrictions assumptions and introducing other variables has developed many theories and emprirical researches that postulate the relevance of the firm's capital structure on its value.

The trade-off theory (Kraus \& Litzenberger, 1973) tries to find the "optimal capital structure" by balancing benefits and costs of the debt with regard mainly to the tax shield and bankruptcy costs. Usually in this context also the agency costs of equity and debt are considered (Morellec et al., 2010, 2004). Based on the agency theory (Jensen, 1986; Jensen \& Meckling, 1976) debt has positive effect on agency cost of equity, reducing the conflicts between shareholders and management, due to its discipline effect on management (Jensen, 1986), but, at the same time, also a negative effect on agency cost of debt, increasing the conflicts between shareholders and debtholders due to the moral hazard and asset-substitutions.

Other theories assume that the "optimal capital structure" is not due to the trade-off between benefits and costs of debt. The pecking order theory (Baker \& Wurgler, 2002; Fama \& French, 2002, 1998; Shyam-Sunder \& Myers, 1999; Myers, 2001, 1984; Myers \& Majluf, 1984) argues that the firm's choices about capital structure are based on a source hierarchy: firm prefers first internal sources and if external one are required, debt is preferred to equity. Also the market time theory (Baker \& Wurgler, 2002) argues that the firm's choices about capital structure are due to the capital market conditions and the manager decisions over time. Firm's capital structure evolves as the cumulative outcome of past attempts to time the equity market (Frank \& Goyal, 2009; Hovakimian, 2006; Ritter, 2003; Baker \& Wurgler, 2002; Baker \& Wurgler, 2002; Korajczyk et al., 2003; Myers \& Majluf, 1984).

The empirical researches have highlighted many determinants, in addition to the models, that could affect the capital structure choices (Frank \& Goyal, 2009, 2003; Rajan \& Zingales, 1995; Harris \& Raviv, 1991; Titman \& Wessels, 1988). The problem is due to their identification and their effects (positive or negative) on the capital structure. It is not unusual that a single determinant has a positive impact on the capital structure choices in some studies while negative in others.

Theories and empirical researches seem to explain some aspects under certain condition of the firm's behaviour. Actually there is still no theory that can fully explain the firm's behaviour on capital structure or, even more, be 
able to define the optimal capital structure.

This paper is a part of the debate. It draws on the most recent literature about the relevance of the economic, financial and institutional conditions of the country on the firm debt choices (Fan et al., 2012; Frank \& Goyal, 2009; Beck et al., 2002; Booth et al., 2001; Demirguc-Kunt \& Maksimovic, 1999). The studies show that the economic and financial development of the country and the quality of its institutional system affect the firm's debt level choices. These effects are usually considered separately. The originality of the paper lies in considering jointly these effects. In this sense, the paper's theoretical hypothesis is that the firm's choices about debt level are functions of several previsions over time related, on the one hand, the firm's characteristics and its expected performances and, on the other hand, the general development of the economy, capital markets and institutional system of country's reference.

This theoretical hypothesis is tested using a panel data of non-financial listed firms in G-7 countries (USA, UK, Canada, Japan, Germany, France and Italy) in the period 1994-2013.

The paper is organized as follows. Section 2 discusses the theoretical background in which it is made explicit the research theoretical hypothesis and its limitations. Section 3 discusses the analysis metholodogy and the dataset. Section 4 presents and discusses the analysis results. Section 5 draws the conclusions of the study.

\section{The Theoretical Background}

In literature many studies have focused on the identification and analysis of the main determinants that affects the firm's choices about debt level. The well-known determinants are the firm asset, size, growth, risk, financial deficit and surplus, profitability, stock price, taxation and the debt level of the industry's reference (Denis \& McKeon, 2012; DeAngelo \& Roll, 2011; Muzir, 2011; Rauh \& Sufi, 2010; Frank \& Goyal, 2003, 2009; Lemmon et al., 2008; De Jong et al., 2008; Byoun, 2008; Kayhan \& Titman, 2007; Brounen at al., 2006; Claessens \& Klapper, 2005; Welch, 2004; Bancel \& Mittoo, 2004; Hall et al., 2004; Graham \& Harvey, 2001; Rajan \& Zingales, 1995; Harris \& Raviv, 1991). These determinants are functions of the firm's structure and characteristics. Recent studies have expanded the perspective. They have investigated the effects of the economic, financial and institutional system of the country's reference on the firm's debt choices (Fan et al., 2012; Frank \& Goyal, 2009; Masulis \& Nahata, 2009; De Jong et al., 2008; Davydenko \& Franks, 2008; Dinc, 2005; Degryse \& Ongena, 2005; Johnson \& Mitton, 2003; Beck et al., 2002; Djankov et al., 2002; La Porta et al., 2002; Booth et al., 2001; Fisman, 2001; Demirguc-Kunt \& Maksimovic, 1999).

The originality of the paper lies in considering all these determinants jointly. The paper's theoretical hypothesis is that firms' debt level choices are based not only on its characteristics and performances, but also on the economic, financial and institutional system of country's references. Therefore, firm has to define and evaluate its debt level sustainability over time based on the systemic and dynamic combination of three main variables:

1) Firm Fundamentals (FF), that groups determinants that affect the firm's debt level choices and refers to its structural characheristics and performances over time. In this context the determinants used are: the firm asset, size, growth, risk, financial deficit and surplus, profitability, stock price, taxation and the debt level of the industry's reference. Therefore the variable groups determinants that are endogenous to the firm and thus under its control;

2) Economic and Financial Conditions (EFC), that groups determinants that affect the firm's debt level choices and refers to the economic and capital markets performances of the country's reference of the firm. In this context, country's economic performances refer to the GDP and inflations rate. The first, has impact on the domestic consumptions, then on the domestic demand, and thus on the firm's ability to develop in the country over time. The second, has effects on the firm's capability to face debt obligation making it less onerous during inflation periods while lot in deflation one. The capital market and bank sector development of the country's reference affect the firm's choices about debt level, because they define the access conditions to debt in terms of amount, time and cost. Greater is the capital market and the bank sector development, greater is the capital movements, and easier is the access to the debt at favorable conditions by competitive firms. Therefore the variable groups determinants are exogenous to the firm and then out of its control. Thus, the firm can only try to manage their effects over time;

3) Institutional Conditions (IC), that groups determinants that affect firm's choices about debt level and refers to the quality and efficiency of legal, bureaucracy and taxation system of the country's reference of the firm. These significantly affect the investors' choices, both in equity and debt, due to the protection of their rights, semplicity of their actions and the weight of overall tax burden. More efficient is the legal system, lower is the bureaucracy and lower is the corporate and personal taxes, greater is the investors' attention on the 
competitive firms. Therefore higher is the competitive firms capability to raise capital if their performances are in line with investors' expectations. Also in this case, the variable groups determinats are exogenous to the firm and then out of its control. Therefore in this case too, the firm can only try to manage their effects over time.

The empirical model proposed has limitations due to some assumptions made that it is necessary to say immediately. These can generate potential sources of bias. The main are the following:

a) it is used a linear model. It is easy to use although not able to grasp the complexity of the problem about debt level;

b) it is assumed that the capital structure's choices of the firm reflect its choices about leverage. Also it is assumed that the leverage refers to the firm's financial debt (both short, medium and long-term). Finally, it is omitted the specification "financial debt" that becomes simply "debt level" of the firm;

c) the total debt of the firm is measured by the ratio of Total Debt (both short, medium and long term) / Total Assets (book value);

d) the firm's debt level is defined based on its book-value. In literature some scholars advocate the book-leverage while others the market-value (Welch, 2004; Myers, 2001, 1984; Shyaman-Sunder \& Myers, 1999; Titman \& Wessels, 1988; Rajan \& Zingales, 1995). Two are the main reasons for the use of market-value: i) the book value is primarily a plug number used to balance the left-hand side and the right-hand side of the balance sheet rather than a managerially relevant number; ii) the book-value is backward-looking by measuring what has taken place while market-value is forward-looking by measuring what will be on the base of expectations and market perspectives. Despite these strong arguments, in this paper it is used book value based on equally strong arguments. Among these, the main are the following: i) market-value of the firm is difficult to determinate in each moment, subject to the market volatility and the data choices by reference to the market value is arbitrary; ii) managers tend to think in term of book value rather than market value because it is more easily accessible, more accurately recorded and not subject to market volatility; iii) the measurement of the firm's economic performances are usually based on income statements and the book value measure of leverage is considered as best proxy of market value; iv) debt is better supported by asset in place than by growth opportunities; v) the main cost of debt is the expected cost of financial distress in the bankruptcy event. In this case the accurate measure of debtholders' liabilities is the book value of debt and not of market value;

e) the dataset are based only on firms reported in Datastream, listed since 1994 and until 2013. With regard to the sample two basic assumptions are needed. First, the percentage of listed firms differs widely across the G-7 countries as well as the average size of firms listed. Also, in each of the G-7 country listed firms may represent only a small part of all firms in the country. But if it is assumed that common institutions in a country can influence both the listed firms and non-listed firms, the information gathered from an analysis of listed firms will have broader implications (Rajan \& Zingales, 1995). Second, it is possible that the firms between the G-7 countries are not perfectly homogeneous because there are not perfectly homogeneous the international data despite the use of a international database as data source;

f) the sample is made from many observations. Material errors can be possible. Also, in order to manage exceptional value for each determinant fluctuation bands allowed are defined. These are built in such a way to consider outliers the values out of band in reasonable way. However the data that are not considered in the analysis do not change the results significantly;

g) there are restrictive assumptions on some determinants. First, it is assumed that the development of bond market is equal to the stock market one. Therefore the development of the stock market can be used as a proxy of the development of the equity and bond market. Second, the determinant legal and bureaucracy system is measured by an index called "Institutional conditions - composit index". It is defined based on information cointained in the Busing Doing annual report of The World Bank. In details, for each year it is calculated as a sum of Strength of legal rights index, Depth of credit information index, Strength of investor protection index, Enforcing Contracts - Time, Enforcing Contracts - Cost, Resolving Insolvency - Time, Resolving Insolvency - Cost, Resolving Insolvency - Recovery rate. For the year data notavailable, they are assumed equal to the data of the first year available;

h) each determinant is usually measured by more than one measuring-indicator. The determinant's sign of correlation (positive or negative) is defined based on the prevailing correlation sign of the joint observation of its measuring-indicators. 


\section{Dataset and Methodology}

Based on the theoretical hypothesis and limitations as discussed in the previous Section, the firm's debt level is function of the three variables as following:

$$
D_{i, t}=f\left(F F_{i, t} ; E F C_{i, t} ; I C_{i, t}\right)
$$

where: $\mathrm{D}_{\mathrm{i}, \mathrm{t}}$ is the debt level of the $\mathrm{i}$-firm in $\mathrm{t}$-time and it is the dependent variable; $\mathrm{FF}_{\mathrm{i}, \mathrm{t}}$ is the variable Firm Fundaments for the i-firm in t-time; $\mathrm{EFC}_{\mathrm{i}, \mathrm{t}}$ is the variable Economic and Financial Conditions for the i-firm in $\mathrm{t}$-time; $\mathrm{IC}_{\mathrm{i}, \mathrm{t}}$ is the variable Institutional Conditions for the $\mathrm{i}$-firm in t-time.

In this context, the determinants grouped in the variable Firm Fundamentals (FF) are the following: Asset (TA), Industry (I), Size (S), Growth (G), Stock Price (SP), Profitability (P), Financial Deficit-Surplus (FDS), Risk (R) and corporate tax (TC). The determinants grouped in the variable Economic and Financial Context (EFC) are the following: Bank sector conditions (BS), Stock market conditions (SM) and Macroeconomic conditions (MC). Finally, the determinants grouped in the variable Institutional Conditions (IC) are the following: Legal and bureaucracy system (LS) and Taxations system (TS).

Based on a panel data, the empirical regression model is the following:

$$
\begin{gathered}
D_{i, t}=\alpha+\beta_{1} F D S_{i, t}+\beta_{2} \cdot P_{i, t}+\beta_{3} \cdot R_{i, t}+\beta_{4} \cdot G_{i, t}+\beta_{5} \cdot T A_{i, t}+\beta_{6} \cdot T C_{i, t}+\beta_{7} \cdot S_{i, t}+ \\
\beta_{8} \cdot S P_{i, t}+\beta_{9} \cdot I_{i, t}+\beta_{10} \cdot B S_{i, t}+\beta_{11} \cdot S M_{i, t}+\beta_{12} \cdot M C_{i, t}+\beta_{13} \cdot L S_{i, t}+\beta_{14} \cdot T S_{i, t}+\varepsilon_{i, t}
\end{gathered}
$$

Each of the determinant grouped in the three variables, is measured by several measuring-indicators as reported

\begin{tabular}{|c|c|c|}
\hline Variable & Determinant & Masuring-Indicator \\
\hline Dependente variable & Debt level (D) & Total Debt (short, medium and long term) / Total Asset \\
\hline \multirow[t]{22}{*}{ Firm Fundamentals (FF) } & \multirow[t]{2}{*}{ Asset (TA) } & TA.1: Tangible Asset / Total Asset \\
\hline & & TA.2: Annual percentage change of total asset \\
\hline & \multirow[t]{2}{*}{ Industry (I) } & I.1: Average industry ratio of Total Debt / Total Capital \\
\hline & & I.2: Average industry ratio of Net Debt / Total Capital \\
\hline & \multirow[t]{2}{*}{ Size (S) } & S.1: natural logaritm of Revenues \\
\hline & & S.2: natural logaritm of Total Asset \\
\hline & Growth $(\mathrm{G})$ & G.1: Enterprice value / Total Asset \\
\hline & \multirow[t]{3}{*}{ Stock Price (SP) } & SP.1: Price volatility \\
\hline & & SP.2: Price / Book Value \\
\hline & & SP.3: Price / Earnings \\
\hline & \multirow[t]{4}{*}{ Profitability (P) } & P.1: ROI \\
\hline & & P.2: ROA \\
\hline & & P.3: ROE \\
\hline & & P.4: annual percentage change of Enterproce Value \\
\hline & \multirow[t]{3}{*}{ Financial Deficit-Surplus (FDS) } & FDS.1: Net Cash Flow Investing / EBIT \\
\hline & & FDS.2: Net Cash Flow Operating / EBIT \\
\hline & & FDS.3: Net Cash Flow Financing / EBIT \\
\hline & \multirow[t]{3}{*}{ Risk (R) } & R.1: EBITDA / Interest Expenses on Debt \\
\hline & & R.2: EBIT / Interest Expenses on Debt \\
\hline & & R.3: EBIT / (EBIT - Interest Expenses on Debt) \\
\hline & \multirow[t]{2}{*}{ Firm Taxation (TC) } & TC.1: Corporate tax rate \\
\hline & & TC.2: Income Tax / Net income before preferred dividend \\
\hline \multirow{8}{*}{$\begin{array}{l}\text { Economic and Financial } \\
\text { Conditions (EFC) }\end{array}$} & \multirow[t]{3}{*}{ Bank Sector Conditions (BS) } & BS.1: Domestic credit to private sector / GDP \\
\hline & & BS.2: Domestic credit to private sector by banks / GDP \\
\hline & & BS.3: Loans to residents / GDP \\
\hline & \multirow[t]{3}{*}{ Stock Market Conditions (SM) } & SM.1: Market capitalization of listed company / GDP \\
\hline & & SM.2: number of listed company \\
\hline & & SM.3: Annual percentage change of S\&P global equity indices \\
\hline & \multirow[t]{2}{*}{ Macroeconomic conditions (MC) } & MC.1: Annual percentage change of the GDP \\
\hline & & MC.2: Annal inflation rate \\
\hline
\end{tabular}
in Table 1.

Table 1. Variables, determinants and measuring-indicators 


\begin{tabular}{|c|c|c|c|}
\hline \multirow{2}{*}{$\begin{array}{l}\text { Institutional } \\
\text { (IC) }\end{array}$} & Conditions & Legal Bureaucracy System (LS) & LS.0: Institutional conditions - composit index \\
\hline & & Taxation System (TS) & $\begin{array}{l}\text { TS.1: Total tax rate } \\
\text { TS.2: Timing of paying taxes }\end{array}$ \\
\hline
\end{tabular}

The sample is a panel data of non-financial firms listed in the G-7 countries (USA, UK, Canada, Japan, Germany, France, Italy) in a period 1994-2013. The choice of G-7 countries is mainly due to the fact that they are the major industrialized countries (Rajan \& Zingales, 1995) with differences in the economic, financial and institutional system. The choice of long period, from 1994 to 2013, is mainly due because the choices about debt level required a long period in order to be fully implemented.

The data source is database Datastream. Other informations are obtained by the World Bank, Organisation for Economic Co-operation and Development (OECD), European Central Bank (ECB), International Moneraty Found (IMF), Eurostat.

The dataset include 4.142 firms for a total observationsof 82.840 as following: 1.220 firms in USA (24.400 observations); 305 firms in UK (6.100 observations); 613 firms in Canada (12.260 observations); 1.616 firms in Japan (32.320 observations); 194 firms in Germany (3.880 observations); 159 firms in France (3.180 observations) and 35 firms in Italy (700 observations). The Table 2 reports the firms included in the dataset Datastream distinguished by industry.

Table 2. Dataset

\begin{tabular}{|c|c|c|c|c|c|c|c|c|}
\hline Industry & USA & UK & Canada & Japan & Germany & France & Italy & Tot \\
\hline Aerospace \& Defense & 41 & 7 & 5 & 4 & 1 & 7 & 1 & 66 \\
\hline Alternative energy & 5 & & & & & & & 5 \\
\hline Automobiles \& parts & 13 & 1 & 4 & 88 & 9 & 9 & 4 & 128 \\
\hline Beverage & 12 & 5 & 5 & 12 & 12 & 6 & & 52 \\
\hline Chemicals & 52 & 9 & 4 & 135 & 10 & 5 & & 215 \\
\hline Construction \& Materials & 50 & 18 & 12 & 197 & 18 & 13 & 8 & 316 \\
\hline Electricity & 37 & 2 & 7 & 10 & 3 & 3 & & 62 \\
\hline Electronic \& electrical equipment & 113 & 16 & 7 & 143 & 8 & 7 & & 294 \\
\hline Fixed line telecommunications & 9 & 2 & 1 & 1 & & & 1 & 14 \\
\hline Food \& drug retailers & 14 & 5 & 6 & 35 & 2 & 4 & & 66 \\
\hline Food producers & 37 & 11 & 8 & 95 & 10 & 10 & & 171 \\
\hline Forestry \& paper & 8 & 1 & 5 & 10 & 2 & 2 & & 28 \\
\hline Gas, water \& multiutilities & 34 & 3 & 3 & 13 & 5 & 2 & 2 & 62 \\
\hline General industrials & 28 & 8 & 4 & 30 & 3 & 4 & 2 & 79 \\
\hline General retailers & 72 & 19 & 10 & 68 & 9 & 6 & 1 & 185 \\
\hline Health care equipment $\&$ service & 96 & 4 & 7 & 20 & 9 & 1 & & 137 \\
\hline Household goods \& home construction & 54 & 17 & 1 & 53 & 14 & 4 & 2 & 145 \\
\hline Industrial engineering & & 25 & 9 & 209 & 32 & 6 & 1 & 282 \\
\hline Industrial metals \& mining & 17 & 1 & 38 & 55 & 4 & 3 & 1 & 119 \\
\hline Industrial transportation & 27 & 6 & 4 & 55 & 5 & 5 & 1 & 103 \\
\hline Leisure goods & 15 & 4 & & 23 & 1 & 3 & & 46 \\
\hline Media & 34 & 23 & 10 & 17 & 2 & 10 & 4 & 100 \\
\hline Mining & 10 & 8 & 328 & 4 & 3 & 2 & & 355 \\
\hline Mobile telecommunications & 3 & 1 & 2 & 1 & & & & 7 \\
\hline Oil \& gas producers & 53 & 7 & 74 & 8 & & 3 & & 145 \\
\hline Oil equipment \& services & 33 & 4 & 13 & 1 & & 2 & 1 & 54 \\
\hline Personal goods & 21 & 7 & 1 & 72 & 14 & 10 & 2 & 127 \\
\hline Pharmaceuticals \& biotechnology & 66 & 5 & 10 & 32 & & 3 & 1 & 117 \\
\hline Software \& computer services & 47 & 13 & 10 & 30 & 1 & 9 & & 110 \\
\hline Support services & 69 & 49 & 12 & 52 & 2 & 6 & 1 & 191 \\
\hline Technology hardware \& equipment & 96 & 7 & 6 & 73 & 4 & 4 & & 190 \\
\hline Tobacco & 3 & 1 & & & & & & 4 \\
\hline Travel \& leisure & 51 & 16 & 7 & 70 & 11 & 10 & 2 & 167 \\
\hline Total Firms & 1.220 & 305 & 613 & 1.616 & 194 & 159 & 35 & 4.142 \\
\hline Number of observations & 24.400 & 6.100 & 12.260 & 32.320 & 3.880 & 3.180 & 700 & 82.840 \\
\hline
\end{tabular}


The Table 3 reports the descriptive statistics of the measuring-indicators of the determinants grouped in the three variables.

Table 3. Descriptive statistics

\begin{tabular}{|c|c|c|c|c|c|c|c|c|c|c|c|c|c|c|}
\hline & \multicolumn{2}{|c|}{ USA } & \multicolumn{2}{|c|}{ UK } & \multicolumn{2}{|c|}{ Canada } & \multicolumn{2}{|c|}{ Japan } & \multicolumn{2}{|c|}{ Germany } & \multicolumn{2}{|c|}{ France } & \multicolumn{2}{|c|}{ Italy } \\
\hline & Mean & Std.Dev & Mean & Std.Dev & Mean & Std.Dev & Mean & Std.Dev & Mean & Std.Dev & Mean & Std.Dev & Mean & Std.Dev \\
\hline D & 0,22 & 0,21 & 0,18 & 0,16 & 0,08 & 0,16 & 0,24 & 0,19 & 0,19 & 0,18 & 0,21 & 0,15 & 0,27 & 0,14 \\
\hline FDS. 2 & 0,79 & 1,98 & 0,81 & 1,97 & 0,39 & 1,48 & 0,82 & 2,29 & 0,63 & 1,83 & 0,78 & 1,79 & 0,83 & 1,66 \\
\hline FDS. 3 & $-0,12$ & 2,13 & $-0,18$ & 2,14 & $-0,47$ & 2,18 & $-0,29$ & 2,04 & $-0,08$ & 1,64 & $-0,05$ & 1,61 & $-0,12$ & 1,72 \\
\hline P. 2 & 2,00 & 20,87 & 5,08 & 13,07 & $-8,29$ & 25,92 & 1,63 & 5,03 & 3,16 & 11,25 & 3,89 & 7,08 & 2,27 & 4,48 \\
\hline P. 3 & 5,57 & 29,32 & 10,90 & 26,85 & $-7,96$ & 28,95 & 2,24 & 14,44 & 4,75 & 27,19 & 7,71 & 16,97 & 2,01 & 19,09 \\
\hline P. 4 & 0,15 & 0,55 & 0,12 & 0,50 & 0,10 & 0,59 & 0,01 & 0,45 & 0,06 & 0,42 & 0,10 & 0,47 & 0,06 & 0,52 \\
\hline R. 1 & 13,26 & 32,62 & 17,55 & 31,51 & $-3,24$ & 30,46 & 25,75 & 36,02 & 13,90 & 27,48 & 17,05 & 24,86 & 8,80 & 12,98 \\
\hline R. 2 & 9,10 & 31,67 & 13,15 & 30,73 & $-5,49$ & 30,81 & 18,29 & 34,59 & 9,47 & 25,39 & 11,77 & 23,36 & 5,00 & 12,15 \\
\hline TA. 2 & 0,10 & 0,34 & 0,09 & 0,29 & 0,12 & 0,47 & 0,01 & 0,12 & 0,05 & 0,24 & 0,07 & 0,23 & 0,03 & 0,27 \\
\hline TC.1 & 23,62 & 18,73 & 22,91 & 16,82 & 5,86 & 13,88 & 34,17 & 23,18 & 20,63 & 20,88 & 25,89 & 18,25 & 26,00 & 22,39 \\
\hline TC. 2 & 0,46 & 1,51 & 0,44 & 1,80 & 0,18 & 1,45 & 0,96 & 2,89 & 0,52 & 2,39 & 0,63 & 2,14 & 1,15 & 5,36 \\
\hline S.1 & 5,38 & 1,58 & 5,10 & 1,40 & 1,64 & 2,47 & 7,32 & 1,50 & 4,69 & 2,12 & 5,56 & 1,52 & 5,80 & 1,05 \\
\hline S.2 & 5,47 & 1,47 & 5,15 & 1,29 & 2,69 & 2,47 & 7,33 & 1,50 & 4,69 & 2,06 & 5,56 & 1,62 & 6,02 & 1,05 \\
\hline SP.1 & 30,54 & 13,94 & 26,93 & 11,95 & 19,14 & 21,88 & 25,22 & 11,39 & 20,40 & 13,13 & 21,23 & 11,96 & 27,58 & 7,84 \\
\hline SP. 2 & 2,46 & 2,85 & 2,22 & 2,76 & 1,25 & 2,66 & 1,22 & 1,30 & 1,88 & 2,45 & 1,59 & 1,72 & 1,44 & 1,32 \\
\hline SP. 3 & 12,45 & 28,43 & 11,63 & 21,41 & $-0,65$ & 20,64 & 19,65 & 34,25 & 13,04 & 29,09 & 13,28 & 22,30 & 12,42 & 28,04 \\
\hline SM. 2 & 6047,40 & 1592,48 & 2248,75 & 278,03 & 2798,80 & 1211,21 & 2997,35 & 542,19 & 687,60 & 118,77 & 794,70 & 146,02 & 273,20 & 21,52 \\
\hline SM.3 & 8,99 & 18,80 & 5,91 & 19,52 & 10,33 & 24,51 & 4,84 & 26,24 & 11,56 & 25,57 & 6,73 & 22,40 & 6,19 & 24,98 \\
\hline MC.1 & 2,57 & 1,77 & 2,36 & 2,22 & 2,65 & 1,67 & 0,96 & 2,07 & 1,40 & 1,92 & 1,56 & 1,49 & 0,74 & 2,06 \\
\hline MC.2 & 2,43 & 0,92 & 2,18 & 0,89 & 1,83 & 0,74 & $-0,02$ & 0,73 & 1,60 & 0,60 & 1,58 & 0,63 & 2,51 & 1,03 \\
\hline LS. 0 & 108,15 & 2,07 & 131,77 & 5,20 & 121,84 & 0,60 & 138,62 & 2,52 & 105,81 & 2,37 & 77,76 & 1,03 & 106,18 & 12,75 \\
\hline TS.1 & 46,04 & 0,41 & 34,83 & 0,45 & 43,58 & 7,49 & 52,16 & 1,76 & 47,41 & 1,13 & 64,99 & 0,18 & 75,62 & 4,85 \\
\hline TS.2 & 289,90 & 60,84 & 106,00 & 2,00 & 120,80 & 4,29 & 326,00 & 16,25 & 198,75 & 6,91 & 132,00 & 0,00 & 328,35 & 22,07 \\
\hline
\end{tabular}

\section{Analysis Results}

The Table 4 reports the regression analysis results. Only significant value of coefficient estimate are reported.

Table 4. Regression analysis results

\begin{tabular}{llllllll}
\hline & USA & JAPAN & UK & CANADA & GERMANY & ITALY & FRANCE \\
\hline FDS.1 & $2,224 \mathrm{e}-03 * *$ & $2,421 \mathrm{e}-03 * * *$ & $2,781 \mathrm{e}-03^{* *}$ & $2,446 \mathrm{e}-03 * *$ & & $4,361 \mathrm{e}-03$. & $2,963 \mathrm{e}-03 *$ \\
& $(7,333 \mathrm{e}-04)$ & $(4,915 \mathrm{e}-04)$ & $(1,068 \mathrm{e}-03)$ & $(8,404 \mathrm{e}-04)$ & & $(2,545 \mathrm{e}-03)$ & $(1,438 \mathrm{e}-03)$ \\
FDS.2 & $-2,224 \mathrm{e}-03 * *$ & & & & & \\
& $(7,521 \mathrm{e}-04)$ & & & & & \\
FDS.3 & $3,406 \mathrm{e}-03 * * *$ & & $3,270 \mathrm{e}-03 * *$ & & $4,379 \mathrm{e}-03 *$ & \\
& $(7,104 \mathrm{e}-04)$ & & $(1,051 \mathrm{e}-03)$ & & $(1,860 \mathrm{e}-03)$ & \\
P.1 & $9,704 \mathrm{e}-04 * * *$ & $2,640 \mathrm{e}-03 * * *$ & $-7,806 \mathrm{e}-04 * * *$ & & $-5,188 \mathrm{e}-04 *$ & $3,760 \mathrm{e}-03$. & \\
\hline
\end{tabular}




\begin{tabular}{|c|c|c|c|c|c|c|c|}
\hline & $(1,210 \mathrm{e}-04)$ & $(3,919 \mathrm{e}-04)$ & $(1,905 e-04)$ & & $(2,444 \mathrm{e}-04)$ & $(2,169 \mathrm{e}-03)$ & \\
\hline P. 2 & $\begin{array}{l}-2,712 \mathrm{e}-03 * * * \\
(1,197 \mathrm{e}-04)\end{array}$ & $\begin{array}{l}-1,431 \mathrm{e}-03 * * \\
(5,308 \mathrm{e}-04)\end{array}$ & $\begin{array}{l}1,073 \mathrm{e}-03 * * * \\
(2,673 \mathrm{e}-049\end{array}$ & & $\begin{array}{l}1,400 \mathrm{e}-03 * * * \\
(3,058 \mathrm{e}-04)\end{array}$ & & $\begin{array}{l}-2,246 \mathrm{e}-03 * * * \\
(5,660 \mathrm{e}-04)\end{array}$ \\
\hline P. 3 & $\begin{array}{l}6,199 \mathrm{e}-04 * * * \\
(6,674 \mathrm{e}-05)\end{array}$ & $\begin{array}{l}-1,123 \mathrm{e}-03 * * * \\
(7,917 \mathrm{e}-05)\end{array}$ & $\begin{array}{l}1,847 \mathrm{e}-04 \\
(1,108 \mathrm{e}-04)\end{array}$ & $\begin{array}{l}-8,123 \mathrm{e}-04 * * * \\
(1,098 \mathrm{e}-04)\end{array}$ & $\begin{array}{l}-3,406 \mathrm{e}-04^{*} \\
(1,330 \mathrm{e}-04)\end{array}$ & $\begin{array}{l}-2,005 \mathrm{e}-03 * * * \\
(4,070 \mathrm{e}-04)\end{array}$ & $\begin{array}{l}-3,297 \mathrm{e}-04 \\
(1,958 \mathrm{e}-04)\end{array}$ \\
\hline P. 4 & $\begin{array}{l}-4,513 \mathrm{e}-03 \\
(2,393 \mathrm{e}-03)\end{array}$ & $\begin{array}{l}-8,242 \mathrm{e}-03 * * * \\
(1,849 \mathrm{e}-03)\end{array}$ & $\begin{array}{l}-8,237 \mathrm{e}-03^{*} \\
(3,930 \mathrm{e}-03)\end{array}$ & & & & \\
\hline R.1 & $\begin{array}{l}-4,033 \mathrm{e}-03 * * * \\
(1,181 \mathrm{e}-04)\end{array}$ & $\begin{array}{l}-3,308 \mathrm{e}-03 * * * \\
(5,810 \mathrm{e}-05)\end{array}$ & $\begin{array}{l}-3,513 \mathrm{e}-03 * * * \\
(2,078 \mathrm{e}-04)\end{array}$ & $\begin{array}{l}-1,250 \mathrm{e}-03 * * * \\
(1,119 \mathrm{e}-04)\end{array}$ & $\begin{array}{l}-5,029 \mathrm{e}-03^{* * *} \\
(2,706 \mathrm{e}-04)\end{array}$ & $\begin{array}{l}-5,233 \mathrm{e}-03^{* * *} \\
(6,524 \mathrm{e}-04)\end{array}$ & $\begin{array}{l}-3,873 \mathrm{e}-03 * * * \\
(2,283 \mathrm{e}-04)\end{array}$ \\
\hline R.2 & $\begin{array}{l}2,901 \mathrm{e}-03 * * * \\
(1,228 \mathrm{e}-04)\end{array}$ & $\begin{array}{l}1,101 \mathrm{e}-03 * * * \\
(6,079 \mathrm{e}-05)\end{array}$ & $\begin{array}{l}1,832 \mathrm{e}-03 * * * \\
(2,158 \mathrm{e}-04)\end{array}$ & $\begin{array}{l}1,032 \mathrm{e}-03 * * * \\
(1,101 \mathrm{e}-04)\end{array}$ & $\begin{array}{l}3,054 \mathrm{e}-03 * * * \\
(2,983 \mathrm{e}-04)\end{array}$ & $\begin{array}{l}2,316 \mathrm{e}-03^{* * *} \\
(6,923 \mathrm{e}-04)\end{array}$ & $\begin{array}{l}1,756 \mathrm{e}-03^{* * *} \\
(2,512 \mathrm{e}-04)\end{array}$ \\
\hline R.3 & $\begin{array}{l}1,478 \mathrm{e}-08^{* * *} \\
(2,970 \mathrm{e}-04)\end{array}$ & $\begin{array}{l}1,993 \mathrm{e}-03 * * * \\
(1,945 \mathrm{e}-04)\end{array}$ & $\begin{array}{l}2,699 \mathrm{e}-03^{* * *} \\
(6,658 \mathrm{e}-04)\end{array}$ & & & & \\
\hline G.1 & $\begin{array}{l}3,378 \mathrm{e}-02 * * * \\
(1,692 \mathrm{e}-03)\end{array}$ & $\begin{array}{l}5,822 \mathrm{e}-02 * * * \\
(2,562 \mathrm{e}-03)\end{array}$ & $\begin{array}{l}6,814 \mathrm{e}-02 * * * \\
(2,851 \mathrm{e}-03)\end{array}$ & $\begin{array}{l}2,310 \mathrm{e}-02 * * * \\
(1,793 \mathrm{e}-03)\end{array}$ & $\begin{array}{l}4,087 \mathrm{e}-02 * * * \\
(4,438 \mathrm{e}-03)\end{array}$ & $\begin{array}{l}1,227 \mathrm{e}-01^{* * *} \\
(1,691 \mathrm{e}-02)\end{array}$ & $\begin{array}{l}7,214 \mathrm{e}-02 * * * \\
(5,010 \mathrm{e}-03)\end{array}$ \\
\hline TA.1 & $\begin{array}{l}1,866 \mathrm{e}-01 * * * \\
(5,387 \mathrm{e}-03)\end{array}$ & $\begin{array}{l}3,395 \mathrm{e}-01 * * * \\
(5,276 \mathrm{e}-03)\end{array}$ & $\begin{array}{l}1,014 \mathrm{e}-01 * * * \\
(7,911 \mathrm{e}-039\end{array}$ & $\begin{array}{l}4,048 \mathrm{e}-02 * * * \\
(4,895 \mathrm{e}-03)\end{array}$ & $\begin{array}{l}3,015 \mathrm{e}-01 * * * \\
(1,244 \mathrm{e}-02)\end{array}$ & & $\begin{array}{l}1,742 \mathrm{e}-01 * * * \\
(1,269 \mathrm{e}-02)\end{array}$ \\
\hline TA. 2 & $\begin{array}{l}1,549 \mathrm{e}-02 * * * \\
(3,729 \mathrm{e}-03)\end{array}$ & & & & & & $\begin{array}{l}6,178 \mathrm{e}-02 * * * \\
(1,009 \mathrm{e}-02)\end{array}$ \\
\hline TC.1 & $\begin{array}{l}-1,055 \mathrm{e}-03 * * * \\
(7,605 \mathrm{e}-05)\end{array}$ & $\begin{array}{l}-4,782 \mathrm{e}-04 * * * \\
(4,342 \mathrm{e}-05)\end{array}$ & $\begin{array}{l}-7,364 \mathrm{e}-04 * * * \\
(1,252 \mathrm{e}-04)\end{array}$ & $\begin{array}{l}-1,080 \mathrm{e}-03 * * * \\
(1,183 \mathrm{e}-04)\end{array}$ & & & \\
\hline TC. 2 & $\begin{array}{l}1,744 \mathrm{e}-03 * \\
(7,473 \mathrm{e}-04)\end{array}$ & $\begin{array}{l}2,228 \mathrm{e}-03 * * * \\
(2,829 \mathrm{e}-04)\end{array}$ & & $\begin{array}{l}3,033 \mathrm{e}-03 * * * \\
(7,825 \mathrm{e}-04)\end{array}$ & & & $\begin{array}{l}1,703 \mathrm{e}-03 \\
(9,869 \mathrm{e}-04)\end{array}$ \\
\hline S.1 & $\begin{array}{l}2,574 \mathrm{e}-02 * * * \\
(2,138 \mathrm{e}-03)\end{array}$ & $\begin{array}{l}2,965 \mathrm{e}-02 * * * \\
(3,364 \mathrm{e}-03)\end{array}$ & $\begin{array}{l}3,871 \mathrm{e}-02 * * * \\
(2,938 \mathrm{e}-03)\end{array}$ & $\begin{array}{l}3,684 \mathrm{e}-02 * * * \\
(1,030 \mathrm{e}-03)\end{array}$ & $\begin{array}{l}2,460 \mathrm{e}-02 * * * \\
(3,960 \mathrm{e}-03)\end{array}$ & $\begin{array}{l}3,047 \mathrm{e}-02 * * \\
(9,602 \mathrm{e}-03)\end{array}$ & $\begin{array}{l}-6,815 \mathrm{e}-03^{*} \\
(2,990 \mathrm{e}-03)\end{array}$ \\
\hline S.2 & $\begin{array}{l}9,467 \mathrm{e}-03 * * * \\
(2,284 \mathrm{e}-03)\end{array}$ & $\begin{array}{l}-1,010 \mathrm{e}-02 * * \\
(3,400 \mathrm{e}-03)\end{array}$ & $\begin{array}{l}-9,875 \mathrm{e}-03 * * \\
(3,272 \mathrm{e}-03)\end{array}$ & $\begin{array}{l}5,119 \mathrm{e}-03 * * * \\
(1,294 \mathrm{e}-03)\end{array}$ & & & $\begin{array}{l}3,551 \mathrm{e}-02 * * * \\
(2,884 \mathrm{e}-03)\end{array}$ \\
\hline SP.1 & $\begin{array}{l}1,088 \mathrm{e}-03 * * * \\
(9,281 \mathrm{e}-05)\end{array}$ & $\begin{array}{l}1,481 \mathrm{e}-03 * * * \\
(7,936 \mathrm{e}-05)\end{array}$ & $\begin{array}{l}1,043 \mathrm{e}-03 * * * \\
(1,620 \mathrm{e}-04)\end{array}$ & & $\begin{array}{l}5,741 \mathrm{e}-04 * * \\
(2,033 \mathrm{e}-04)\end{array}$ & $\begin{array}{l}1,255 \mathrm{e}-03^{*} \\
(5,808 \mathrm{e}-04)\end{array}$ & $\begin{array}{l}1,025 \mathrm{e}-03^{* * *} \\
(1,948 \mathrm{e}-04)\end{array}$ \\
\hline SP.2 & $\begin{array}{l}-1,787 \mathrm{e}-02 * * * \\
(4,786 \mathrm{e}-04)\end{array}$ & $\begin{array}{l}1,238 \mathrm{e}-02 * * * \\
(7,602 \mathrm{e}-04)\end{array}$ & $\begin{array}{l}-1,333 \mathrm{e}-02 * * * \\
(7,594 \mathrm{e}-04)\end{array}$ & $\begin{array}{l}-1,107 \mathrm{e}-02 * * * \\
(5,413 \mathrm{e}-04)\end{array}$ & $\begin{array}{l}-1,229 \mathrm{e}-02 * * * \\
(1,134 \mathrm{e}-03)\end{array}$ & $\begin{array}{l}-9,046 \mathrm{e}-03^{*} \\
(4,439 \mathrm{e}-03)\end{array}$ & $\begin{array}{l}-1,776 \mathrm{e}-02 * * * \\
(1,700 \mathrm{e}-03)\end{array}$ \\
\hline SP.3 & $\begin{array}{l}-1,539 \mathrm{e}-04 * * * \\
(4,288 \mathrm{e}-05)\end{array}$ & $\begin{array}{l}-1,937 \mathrm{e}-04 * * * \\
(2,581 \mathrm{e}-05)\end{array}$ & & & $\begin{array}{l}-1,592 \mathrm{e}-04 \\
(8,524 \mathrm{e}-05)\end{array}$ & & $\begin{array}{l}-2,001 \mathrm{e}-04 * \\
(1,009 \mathrm{e}-04)\end{array}$ \\
\hline I.1 & $\begin{array}{l}7,847 \mathrm{e}-02 * * * \\
(1,665 \mathrm{e}-02)\end{array}$ & & $\begin{array}{l}4,559 \mathrm{e}-02 * * \\
(1,472 \mathrm{e}-02)\end{array}$ & $\begin{array}{l}1,044 \mathrm{e}-01 * * * \\
(1,640 \mathrm{e}-02)\end{array}$ & $\begin{array}{l}-4,693 \mathrm{e}-02^{* *} \\
(1,678 \mathrm{e}-02)\end{array}$ & $\begin{array}{l}9,607 \mathrm{e}-02 * * \\
(3,388 \mathrm{e}-02)\end{array}$ & \\
\hline I. 2 & $\begin{array}{l}8,951 \mathrm{e}-02 * * * \\
(1,254 \mathrm{e}-02)\end{array}$ & $\begin{array}{l}7,673 \mathrm{e}-02 * * * \\
(1,160 \mathrm{e}-02)\end{array}$ & $\begin{array}{l}9,412 \mathrm{e}-02 * * * \\
(1,375 \mathrm{e}-02)\end{array}$ & $\begin{array}{l}3,206 \mathrm{e}-02 * * \\
(1,199 \mathrm{e}-02)\end{array}$ & $\begin{array}{l}1,074 \mathrm{e}-01 * * * \\
(1,368 \mathrm{e}-02)\end{array}$ & & $\begin{array}{l}6,969 \mathrm{e}-02 * * * \\
(1,475 \mathrm{e}-02)\end{array}$ \\
\hline BS.1 & & $\begin{array}{l}6,525 \mathrm{e}-04 * \\
(2,876 \mathrm{e}-04)\end{array}$ & & $\begin{array}{l}4,766 \mathrm{e}-04^{*} \\
(1,888 \mathrm{e}-04)\end{array}$ & $\begin{array}{l}-8,377 \mathrm{e}-01 * * \\
(2,819 \mathrm{e}-01)\end{array}$ & & \\
\hline BS. 2 & $\begin{array}{l}-5,617 \mathrm{e}-03 \\
(3,028 \mathrm{e}-03)\end{array}$ & & & $\begin{array}{l}-7,642 \mathrm{e}-04 \\
(4,601 \mathrm{e}-04)\end{array}$ & $\begin{array}{l}8,396 \mathrm{e}-01 * * \\
(2,825 \mathrm{e}-01)\end{array}$ & & \\
\hline BS. 3 & $\begin{array}{l}6,285 \mathrm{e}-01^{*} \\
(2,458 \mathrm{e}-01)\end{array}$ & $\begin{array}{l}-9,149 \mathrm{e}-02 * \\
(3,602 \mathrm{e}-02)\end{array}$ & & & $\begin{array}{l}-3,398 \mathrm{e}-01 \\
(1,940 \mathrm{e}-01)\end{array}$ & & \\
\hline SM.1 & $\begin{array}{l}5,912 \mathrm{e}-04 * \\
(2,808 \mathrm{e}-04)\end{array}$ & $\begin{array}{l}-6,379 \mathrm{e}-04 * * * \\
(7,207 \mathrm{e}-05)\end{array}$ & & $\begin{array}{l}-2,806 \mathrm{e}-04^{* *} \\
(1,055 \mathrm{e}-04)\end{array}$ & & & \\
\hline SM.2 & $\begin{array}{l}-1,300 \mathrm{e}-05^{* *} \\
(4,295 \mathrm{e}-06)\end{array}$ & $\begin{array}{l}-1,390 \mathrm{e}-05 \\
(7,137 \mathrm{e}-06)\end{array}$ & & & & & \\
\hline SM.3 & & $\begin{array}{l}2,136 \mathrm{e}-04 * * * \\
(3,784 \mathrm{e}-05)\end{array}$ & & & & & \\
\hline MC.1 & & $\begin{array}{l}2,108 \mathrm{e}-03 * * * \\
(6,249 \mathrm{e}-04)\end{array}$ & & & & & \\
\hline MC.2 & & $\begin{array}{l}-3,304 \mathrm{e}-03 * * \\
(1,242 \mathrm{e}-03)\end{array}$ & & & & & \\
\hline LS.0 & & $\begin{array}{l}2,020 \mathrm{e}-03 * * * \\
(3,225 \mathrm{e}-04)\end{array}$ & & & $\begin{array}{l}-3,572 \mathrm{e}-03^{*} \\
(1,768 \mathrm{e}-03)\end{array}$ & & \\
\hline TS.1 & & $-3,048 \mathrm{e}-03 * * *$ & & & & & \\
\hline
\end{tabular}




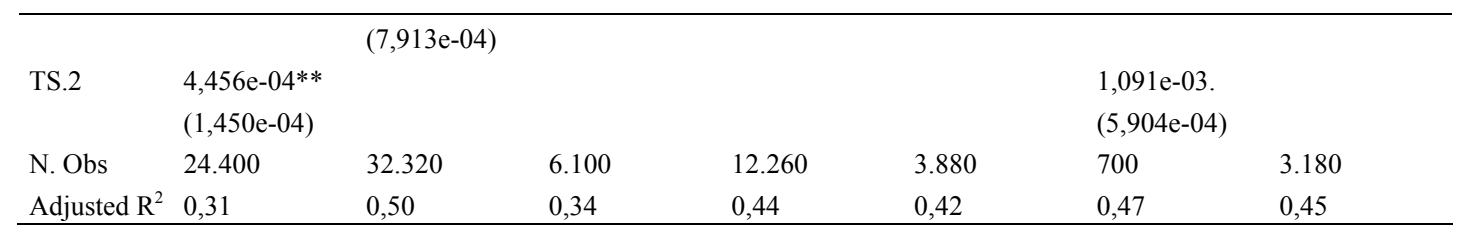

Note. Dependent variable: D. The first number is the estimate and the second in brackets is the standard error.

The coefficient estimate is significantly different from zero at: 0 '***'; $0,001^{\text {'**'? }}$; 0,01 '*’; 0,05 '.'.

The analysis shows that the debt choices for the firms in UK and France are based only on the variable Firm Fundamentals. Otherwise for the firms in USA, Canada, Japan, Germany and Italy, the debt choices are based also on the variable Economic and Financial Conditions and the variable Institutional Conditions with differente relevance.

The determinant Asset (TA) is statistically significant and relevant for the firms in G-7 countries except for the firms in Italy. The analysis founds a positive relationship between firm's debt level and the determinant with regard to its measuring-indicators in all countries. Two are the main reasons: first, debt is a primarily source to finance investment in asset; second, asset are usually used as a collateral debt because provide guarantees to creditors due their liquidity.

The determinant Industry (I) is statistically significant and relevant for the firms in all G-7 countries. The analysis founds a positive relationship between firm's debt level and the determinant with regard to its measuring-indicators in all countries except in part in Germany (with regard I.1). The relationship is explained mainly due to the fact that investments and financing needs tend to be the same in the industry and, thus, the choices about debt level tend to be homogeneus among firms.

The determinant Size (S) is statistically significant and relevant for the firms in all G-7 countries. The analysis founds a positive relationship between the firm's debt level and the determinant with regard its measuring-indicators in all countries. The relationship is explained to the fact that firm's size are usually considered by the investors a good indicator about the firm's capabilities to face debt and its obligations.

The determinant Growth $(\mathrm{G})$ is statistically significant and relevant for the firms in all G-7 countries. The analysis found a positive relationship between firm's debt level and the determinant with regard its measuring-indicators in all country. Two are the main reasons: first, debt is a one of the mainly source to finance the firm's growth over time; second, firm's growth opportunities are a good indicator about the firm's capabilities to face debt and its obbligations.

The determinant Stock Price (SP) is statistically significant and relevant for the firms in all G-7 countries. The analysis found a positive relationship between firm's debt level and its price volatility (SP.1) while negative with ratios price/book value and price/earning (SP.2 and SP.3) in all countries. The positive relationship is due to the fact that the increase (decrease) in price indicates greater (lower) firm's growth prospects and therefore greater (lower) debt capacity. Otherwise the negative relationship could be due to the fact that the higher the price compared to book value and earnings, the greater the manager's propensity to finance the firm's activites through equity rather than debt.

The determinant Profitability (P) is statistically significant and relevant for the firms in all G-7 countries. The analysis finds a positive relationship between firm's debt level and its ROI (P.1) for the firms in USA, Japan and Italy while negative for the firms in UK and Germany. Otherwise there is a positive relationship between debt level and ROA (P.2) for the firms in UK and Germany, while negative for the firms in USA, Japan and France. There is also a positive relationship between debt level and ROE (P.3) for the firms in USA and UK, while negative for the firms in Canada, Japan, Germany, France and Italy. Finally, there is a positive relationship between the debt level and the change in enterprice value (P.4) for the firms in USA and UK. The analysis finds discordant results with regard to the relationship between firm's debt level and its performance in line with the literature. The causes of these findings may be many. One possible interpretation is due to the self-financing on the one hand, and the financing of investment on the other hand. Therefore, the negative relationship is due to the fact that higher are the firm's performances, greater is the self-financing and then lower is the debt level. Otherwise the positive relationship is due to the fact that higher is the debt level, greater are sources to invest and then higher are the firm's performances.

The determinant Financial Deficit and Surplus (FDS) is statistically significant and relevant for the firms in all G-7 countries. The analysis finds a positive relationship between the firm's debt level and its net cash-flow 
investing (FDS.1) and financing (FDS.3) in all countries while it's negative with net cash-flow operating (FDS.2) only for firms in USA where measuring-indicator is statistically significant. The positive relationship is due to the fact that debt is a primarily source to cover firm's financial needs. The negative relationship could be explained considering that for firms in USA the net cash-flow from operations are used to finance the firm's activities reducing its debt reliance.

The determinant Risk (R) is statistically significant and relevant for the firms in all G-7 countries. The analysis finds a positive relationship between the firm's debt level and the ratio of EBIT to interest expenses on debt (R.2 and R.3) in all countries. Therefore, higher is the EBIT compared to interest expenses on debt, higher is the firm's capabilities to cover the cost of debt and, lower is the firm's risk and then greater is the firm debt capabilities. Otherwise the analysis finds a negative relationship between firm's debt level and the ratio of EBITDA to interest expenses on debt (R.1) in all countries except in UK. In this case the negative relationship is not related to the firm's risk but to the firm's self-financing capability due to the high EBITDA.

The determinant Taxation (TC) is statistically significant for the firms in USA, UK, Canada and Japan. In this context the determinant refers to the firm's corporate taxes by distinguished between the income taxes that firm should pay in order to its tax rate (TC.1) and the income taxes that the firm pays on the basis of the fiscal and accounting policies adopted (TC.2). The analysis finds a negative relationship between firm's debt level and its tax rate on income, while a positive relationship with income taxes paid on the bases of its fiscal and accounting policies adopted. The positive relationship is due to the real application of the tax shield on the base of the accounting and fiscal policies adopted by the firm. Otherwise the negative relationship is due to the fact that higher is the tax rate on income, higher is the potential taxes to be paid by firm and greater is the risk of losses on income and then lower is the firm's debt capacity.

The determinant Country Bank Sector Conditions (BS), is statistically significant for the firms in Germany, USA, Japan and Canada. In this context the determinant's correlation sign is defined based on the measuring-indicators' weigh in regression equation. The analysis finds a positive relationship between firm's debt level and determinant in USA while negative in Canada, Japan and Germany. The difference is probably due to the different role of the bank system in firms financing. Therefore, the negative relationship is due to the fact that in bank-oriented economy, the banking system controls about debt level of the firm are rigorous to the limit point, in some cases, to be the bank itself to define the firm's optimal debt level. In this case the bank tends to keep down the debt level of the firm. Otherwise the positive relationship is due to the fact that in market-oriented economy, the bank system finances the firm but the market usually to make judgments about the degree of firm's solvency. This interpretation seems confirmed also by the average level of debt that is higher for the firms in USA, where the correlation is positive, than firms in Canada, Germany and Japan, where the correlation is negative.

The determinant Country Stock Market Conditions (SM), is statistically significant for the firms in USA, Canada and Japan. Also in this case the determinant's correlation sign is defined based on the measuring-indicators' weigh in regression equation. The analysis finds a positive relationship between firm's debt level and determinant in USA while negative in Canada and Japan. In market-oriented economy the relationship is positive where is the market to evaluate the firm and its choices about debt level. Otherwise in bank-oriented economy the relationship tend to be negative where is the bank to evalutate and to define the debt level of the firm.

The determinant Country macroeconomic conditions (MC), is statistically significant only for the firms in Japan. The analysis finds a positive relationship between firm's debt level and the GDP (MC.1) while negative with inflation rate (MC.2). The positive relationship is due to the fact that the GDP growth enables the development of firms and therefore the need for funding sources to support the development. Otherwise the negative relationship is due to the fact that the growth of inflation reduces the value of the debt at the expense of the creditors.

The determinant Country Taxation System is statistically significant for the firms in Japan, Italy and USA. In this context the determinant refers to the taxation system of the country's reference of the firm with regard to the general both debtholders and shareholders personal taxes differently from the determinant taxation (TC) that refers to the firm's corporate taxes. The analysis finds a positive relationship between firm's debt level and the determinant in USA and Italy while negative in Japan. The sign of the correlation is mainly due to the real tax, net of evasion and avoidance, on bondholders and equityholders. Therefore, if the taxation on bondholders is less than equityholders, the relationship is positive otherwise it is negative.

The determinant Country Legality and Bureaucracy System (LS) is statistically relevant for the firms in Japan and Germany. The analysis finds a positive relationship between firm's debt level and the determinant in Japan 
while negative in Germany. It is mainly due to the differences in the institutional system between two countries.

\section{Conclusions}

The theoretical hypothesis of the paper is that the firm's debt level choices are functions of systemic and dynamic combination of previsions over time about, on the one hand, the firm's characteristics and its expected performances and, on the other hand, the economic and capital markets performances and the institutional system quality of the country's reference. Therefore the firm has to define the debt level considering not only the perspectives about firm's fundamentals, related to its characteristics and performances, but also perspectives about economic and capital market performances and institutional system development of the country's reference.

The analysis finds evidence of the theoretical hypothesis in USA, Canada, Japan, Germany and Italy with different relevance while not in UK and France where the firm's debt choices are based only on its fundamentals.

The empirical model proposed is suitable for extensions. It can be improved by identifying new determinants in the three variable and/or differences determinants' measuring-indicators without this changes the logical and methodological approach.

\section{References}

Abor, J. (2005). The effect of capital structure on profitability: Empirical analysis of listed firms in Ghana. Journal of Risk Finance, 6, 438-445. http://dx.doi.org/10.1108/15265940510633505

Baker, M., \& Wurgler, J. (2002). Market timing and capital structure. Journal of Finance, 57, 1-32. http://dx.doi.org/10.1111/1540-6261.00414

Bancel, F., \& Mittoo, U. (2004). Cross-country determinants of capital structure choice: A survey of European firms. Financial Management, 33, 103-132.

Beck, T., Demirgüç-Kunt, A., \& Maksimovic, V. (2002). Financing patterns around the world: The role of institutions. World Bank Policy Research Working Paper 2905.

Berkowitz, D., Pistor, D., \& Richard, J. F. (2003). Economic development, legality, and the transplaneteffect. European Economic Review, 47, 165-195. http://dx.doi.org/10.1016/S0014-2921(01)00196-9

Bhagat, S., Bolton, B., \& Subramanian, A. (2011). Manager characteristics and capital structure: Theory and Evidence. Journal of Financial and Quantitative Analysis, 46, 1581-1627. http://dx.doi.org/10.1017/S0022109011000482

Booth, L., Aivazian, V., Demirgüç-Kunt, A., \& Maksimovic, V. (2001). Capital structure in developing countries. Journal of Finance, 55, 87-130. http://dx.doi.org/10.1111/0022-1082.00320

Brennan, M., \& Kraus, A. (1987). Efficient financing under asymmetric information. Journal of Finance, 42 , 1225-1243. http://dx.doi.org/10.1111/j.1540-6261.1987.tb04363.x

Brounen, D., De Jong, A., \& Koedijk, K. (2006). Capital structure policies in Europe: Survey evidence. Journal of Banking and Finance, 30, 1409-1442. http://dx.doi.org/10.1016/j.jbankfin.2005.02.010

Byoun, S. (2008). How and When Do Firms Adjust Their Capital Structure Toward Targets? Journal of Finance, 63, 3069-3096. http://dx.doi.org/10.1111/j.1540-6261.2008.01421.x

Claessens, S., \& Klapper, L. F. (2005). Bankruptcy around the World: Explanations of Its Relative Use. American Law and Economics Review, 7, 253-283. http://dx.doi.org/10.1093/aler/ahi004

Davydenko, S. A., \& Franks, J. R. (2008). Do Bankruptcy Codes Matter? A Study of Defaults in France, Germany and the UK. Journal of Finance, 63, 565-608. http://dx.doi.org/10.1111/j.1540-6261.2008.01325.x

De Jong, A., Kabir, R., \& Nguyen, T. T. (2008). Capital Structure around the World: the Roles of Firm and Country Specific Determinants. Journal of Banking and Finance, 32, 1954-1969. http://dx.doi.org/10.1016/j.jbankfin.2007.12.034

De Luca, P. (2014). Capital structure and economic performance of the firm: Evidence from Italy. International Journal of Management, 5, 1-20.

DeAngelo, H., \& Roll, R. (2011). How Stable Are Corporate Capital Structure? Working paper, USC Marshall School, Los Angeles.

DeAngelo, H., DeAngelo, L., \& Stulz, R. M. (2010). Seasoned Equity Offerings, Market Timing, and the 
Corporate Ligecycle. Journal of Financial Economics, 95, 275-295. http://dx.doi.org/10.1016/j.jfineco.2009.11.002

DeAngelo, H., DeAngelo, L., \& Whited, T. M. (2011). Capital Structure Dynamics and Transitory Debt. Journal of Finanancial Economics, 99, 235-261. http://dx.doi.org/10.1016/j.jfineco.2010.09.005

Degryse, H., \& Ongena, S. (2005). Distance, Lending Relationship and Competition. Journal of Finance, 55 , 231-266. http://dx.doi.org/10.1111/j.1540-6261.2005.00729.x

Demirgüç-Kant, A., \& Maksimovic, V. (1998). Law, Finance, and Firm Growth. Journal of Finance, 53, 2107-2137. http://dx.doi.org/10.1111/0022-1082.00084

Demirgüç-Kant, A., \& Maksimovic, V. (1999). Institutions, financial markets and firm debt maturity. Journal of Financial Economics, 54, 295-336. http://dx.doi.org/10.1016/S0304-405X(99)00039-2

Denis, J. D., \& McKeon, S. B. (2012). Debt Financing and Financial Flexibility Evidence from Proactive Leverage Increases. The Review of Financial Studies, 25, 1897-1929. http://dx.doi.org/10.1093/rfs/hhs005

Dinc, I. S. (2005). Politicians and Banks: Political Influences on Government-Owned Banks in Emerging Markets. Journal of Financial Economics, 77, 453-479. http://dx.doi.org/10.1016/j.jfineco.2004.06.011

Djankov, S., La Porta, R., Lopez-de-Silanes, F., \& Shleifer, A. (2002). The Regulation on Entry. Quarterly Journal of Economics, 117, 1-37. http://dx.doi.org/10.1162/003355302753399436

Fama, E. F., \& French, K. R. (1998). Taxes, financing decisions, and firm value. Journal of Finance, 53, 819-843. http://dx.doi.org/10.1111/0022-1082.00036

Fama, E. F., \& French, K. R. (2002). Testing trade-off and pecking order predictions about dividends and debt. Review of Financial Studies, 15, 1-33. http://dx.doi.org/10.1093/rfs/15.1.1

Fan, J., Titman, S., \& Twite, G. (2012). An International Comparison of Capital Structure and Debt Maturity Choices. Journal of Financial and Quantitative Analysis, 47, 23-56. http://dx.doi.org/10.1017/S0022109011000597

Fisman, R. (2001). Estimating the Value of Political Connections. American Economic Review, 91, 1095-1102. http://dx.doi.org/10.1257/aer.91.4.1095

Frank, M. Z., \& Goyal, V. K. (2003). Testing the pecking order theory of capital structure. Journal of Financial Economics, 67, 217-248. http://dx.doi.org/10.1016/S0304-405X(02)00252-0

Frank, M. Z., \& Goyal, V. K. (2009). Capital structure decisions: Which factors are reliably important? Financial Management, 38, 1-37. http://dx.doi.org/10.1111/j.1755-053X.2009.01026.x

Giannetti, M. (2003). Do better institutions mitigate agency problems? Evidence from corporate finance choices. Journal of Financial and Quantitative Analysis, 38, 185-212. http://dx.doi.org/10.2307/4126769

Graham, J. R., \& Harvey, C. R. (2001). The theory and practice of corporate finance: Evidence from the field. Journal of Financial Economics, 60, 187-243. http://dx.doi.org/10.1016/S0304-405X(01)00044-7

Hall, G. C., Hutchinson, P. J., \& Michaelas, N. (2004). Determinants of the capital structures of European SMEs. Journal of Business Finance and Accounting, 31, http://dx.doi.org/10.1111/j.0306-686X.2004.00554.x

Harris, M., \& Raviv, A. (1991). The theory of Capital Structure. Journal of Finance, 1, 297-355. http://dx.doi.org/10.1111/j.1540-6261.1991.tb03753.x

Hovakimian, A. (2006). Are observed capital structures determined by equity market timing? Journal of Financial and Quantitative Analysis, 41, 221-243. http://dx.doi.org/10.1017/S0022109000002489

Jensen, M. C. (1986). Agency cost of free cash flow, corporate finance and takeovers. American Economic Review, 76, 323-329.

Jensen, M. C., \& Meckling, W. (1976). Theory of the firm: Managerial behaviour, agency costs, and ownership structure. Journal of Financial Economics, 3, 305-360. http://dx.doi.org/10.1016/0304-405X(76)90026-X

Johnson, S., \& Mitton, T. (2003). Cronyism and Capital Controls: Evidence from Malaysia. Journal of Financial Economics, 67, 351-382. http://dx.doi.org/10.1016/S0304-405X(02)00255-6

Kayhan, A., \& Titman, S. (2007). Firms' Histories and Their Capital Structures. Journal of Financial Economics, 83, 1-32. http://dx.doi.org/10.1016/j.jfineco.2005.10.007

Korajczyk, R. A., \& Levey, A. (2003). Capital Structure Choice: Macroeconomic Conditions and Financial 
$\begin{array}{lllll}\text { Constraints. } & \text { Journal } & \text { Financial } & \text { Economics, } & 68,\end{array}$ http://dx.doi.org/10.1016/S0304-405X(02)00249-0

Kraus, A., \& Litzenberger, R. H. (1973). A State-Preference Model of Optimal Financial Leverage. Journal of Finance, 28, 911-922. http://dx.doi.org/10.1111/j.1540-6261.1973.tb01415.x

La Porta, R., Lopez-de-Silanes, F., Shleifer, A., \& Vishny, R. W. (2002). Investor Protection and Corporate Valuation. Journal of Finance, 57, 1147-1170. http://dx.doi.org/10.1111/1540-6261.00457

Leland, H., \& Pyle, D. (1977). Information asymmetrics, financial structure, and financial intermediation. Journal of Finance, 32, 371-388. http://dx.doi.org/10.2307/2326770

Lemmon, M. L., Roberts, M. R., \& Zender, J. F. (2008). Back to the beginning: Persistence and the cross-section of corporate capital structure. Journal of Finance, 63, 1575-1608. http://dx.doi.org/10.1111/j.1540-6261.2008.01369.x

Miller, M. (1977). Debt and taxes. Journal of Finance, 32, 261-276.

Modigliani, F., \& Miller, M. (1958). The Cost of Capital, Corporation Finance and the Theory of Finance. American Economic Review, 48, 261-297.

Modigliani, F., \& Miller, M. (1963). Corporate income taxes and the cost of capital: A correction. American Economic Review, 53, 433-443.

Morellec, E. (2004). Can managerial discretion explain observed leverage ratios? Review of Financial Studies, 17, 257-294. http://dx.doi.org/10.1093/rfs/hhg036

Morellec, E., \& Schurhoff, N. (2010). Dynamic investment and financing under personal taxation. Review of Financial Studies, 23, 101-146. http://dx.doi.org/10.1093/rfs/hhp062

Muzir, E. (2011). Triangle Relationship among Firm Size, Capital Structure Choice and Economic performance. Some Evidence form Turkey. Journal of Management Research, 11, 87-98.

Myers, S. C. (2001). Capital structure. Journal of Economic Perspectives, 15, 81-102. http://dx.doi.org/10.1257/jep.15.2.81

Myers, S. C., \& Majluf, N. S. (1984). Corporate financing and investment decision when firms have information that investors do not have. Journal of Financial Economics, 13, 187-221. http://dx.doi.org/10.1016/0304-405X(84)90023-0

Myers, S. C. (1984). The capital structure puzzle. Journal of Finance, 39, 575-592. http://dx.doi.org/10.2307/2327916

Opler, T. C., \& Titman, S. (1994). Financial distress and corporate performance. Journal of Finance, 3 , 1015-1040. http://dx.doi.org/10.1111/j.1540-6261.1994.tb00086.x

Rajan, R. G., \& Zingales, L. (1995). What do we know about capital structure: some evidence from international data. Journal of Finance, 50, 1421-1460. http://dx.doi.org/10.1111/j.1540-6261.1995.tb05184.x

Rauh, J., \& Sufi, A. (2010). Capital Structure and Debt Structure. Review of Financial Studies, 23, 4242-4280. http://dx.doi.org/10.1093/rfs/hhq095

Ritter, J. (2003). Investment banking and security issuance. In G. Constantinides, M. Harris, \& R. Stulz (Eds.), Handbook of the Economics of Finance. Amsterdam: Elsevier.

Salehi, M., \& Manesh, N. B. (2012). A Study of the Roels of Firm and Country on Specific Determinates in Capital Structure: Iranian Evidence. International Management Review, 8, 51-62.

Shyam-Sunder, L., \& Myers, S. C. (1999). Testing static tradeoff against pecking order models of capital structure. Journal of Financial Economics, 51, 219-244. http://dx.doi.org/10.1016/S0304-405X(98)00051-8

Simerly, R. L., \& Mingfang, L. (2000). Environmental dynamism, capital structure and performance: A theoretical integration and an empirical test. Strategic Management Journal, 21, 31-49. http://dx.doi.org/10.1002/(SICI)1097-0266(200001)21:1<31::AID-SMJ76>3.0.CO;2-T

Strebulaev, A. I. (2007). Do tests of capital structure theory mean what they say? Journal of Finance, 62, 1747-1787. http://dx.doi.org/10.1111/j.1540-6261.2007.01256.x

Stulz, R. (1990). Managerial discretion and optimal financing policies. Journal of Financial Economics, 26, 3-27. http://dx.doi.org/10.1016/0304-405X(90)90011-N

Titman, S., \& Tsyplakov, S. (2007). A dynamic model of optimal capital structure. Review of Finance, 11, 
401-451. http://dx.doi.org/10.1093/rof/rfm017

Titman, S., \& Wessels, R. (1988). The determinants of capital structure choice. Journal of Finance, 43, 1-19. http://dx.doi.org/10.1111/j.1540-6261.1988.tb02585.x

Welch, I. (2004). Capital Structure and Stock Returns. Journal of Political Economy, 112, 106-131. http://dx.doi.org/10.1086/379933

Wurgler, J. (2000). Financial markets and the allocation of capital. Journal of Financial Economics, 58, $187-214$. http://dx.doi.org/10.1016/S0304-405X(00)00070-2

\section{Copyrights}

Copyright for this article is retained by the author(s), with first publication rights granted to the journal.

This is an open-access article distributed under the terms and conditions of the Creative Commons Attribution license (http://creativecommons.org/licenses/by/3.0/). 